\title{
Relação entre exercício físico, depressão e índice de massa corporal
}

\author{
J. Vasconcelos-Raposo, H.M. Fernandes, M. Mano, E. Martins
}

\begin{abstract}
O objectivo deste estudo é conhecer a relação entre o exercício físico, a depressão e o índice de massa corporal (IMC). A amostra do estudo foi constituída por 175 participantes ( 43 do sexo masculino e 132 do sexo feminino) com idades compreendidas entre os 18 e 27 anos. Os instrumentos utilizados foram o Inventário de Depressão de Beck (BDI), numa versão adaptada e validada para a língua portuguesa, e uma adaptação da escala de exercício físico proposta por Prochaska, Sallis, e Long (2001). Os resultados apontam para um correlação negativa entre o exercício físico e a depressão, com significância estatística. O grupo que não atinge a prática de exercício físico recomendado apresenta valores médios de depressão superiores. Em conclusão, este estudo permitiu confirmar estudos prévios que evidenciaram os efeitos positivos do exercício físico sobre a depressão.
\end{abstract}

Palavras-chave: exercício físico, depressão, índice de massa corporal

\section{Relation between exercise, depression and body mass index}

The purpose of this study is to determine the relationship between physical exercise, depression, and body mass index (BMI). The sample of the study consisted of 175 participants ( 43 male and 132 female) with ages between the 18 and 27 years. The used instruments were: an adapted and validated Portuguese version of the Beck Depressive Inventory (BDI) and an adaptation of the physical exercise scale developed by Prochaska, Sallis and Long (2001). The results suggested a negative correlation between the physical exercise and depression, with statistical significance. The group that does not reach the recommended level of physical exercise presents higher scores of depression in comparison with the group that reaches. This study corroborates previous studies that suggested positive effects of physical exercise on depression.

Key words: exercise, depression, body mass index

Submetido: 19.12.2008 | Aceite: 19.03.2009

José Vasconcelos Raposo. Professor Catedrático de Psicologia da Universidade de Trás-os-Montes e Alto Douro. Membro integrado do CIDESD.

Helder M. Fernandes. Doutor em Psicologia. Docente convidado da Universidade de Trás-os-Montes e Alto Douro. Membro integrado do CIDESD.

Miriam Mano e Edith Martins. Alunas da Licenciatura em Psicologia da Universidade de Trás-os-Montes e Alto Douro.

Endereço para correspondência: Universidade de Trás-os-Montes e Alto Douro, Rua Dr. Manuel Cardona, 5000-558 Vila Real. E-mail: jvraposo@utad.pt 
Ao longo dos últimos anos, verificou-se um aumento do número de sujeitos com quadro clínico de depressão e segundo a Organização Mundial de Saúde (OMS), em 2020, a depressão será das doenças com maior prevalência na população em geral (McKendree-Smith, Floyd, \& Scogin, 2003). Em Portugal, a sintomatologia depressiva é significativamente menos intensa nos estudantes universitários do sexo masculino do que nos estudantes do sexo feminino (Campos \& Gonçalves, 2004).

Segundo o Manual de Diagnóstico e Estatística das Perturbações Mentais (DSM-IVTR) (APA, 2000) a depressão major é descrita como um período de pelo menos duas semanas durante o qual existe humor depressivo ou perda de interesse em quase todas as actividades. Os indivíduos devem, também, experimentar pelo menos um sintoma adicional de uma lista que inclui alterações no apetite ou peso, sono e actividade psicomotora, diminuição da energia, sentimentos de desvalorização pessoal ou culpa, dificuldades em pensar, concentrar-se ou tomar decisões, ou pensamentos recorrentes a propósito da morte ou ideação, planos ou tentativas suicidas.

No que se refere ao exercício físico este pode ser definido como uma forma de actividade física, que corresponde ao conjunto de movimentos corporais planificados, estruturados e repetitivos e à qual o individuo adere com o objectivo de implementar ou manter uma ou mais componentes da condição física e da sua saúde (Buckworth \& Dishman, 2002).

Neste sentido, estudos recentes têm-se debruçado sobre o potencial papel da actividade física na prevenção e/ou tratamento da depressão e dos sintomas depressivos (Paluska \& Schwenk, 2000). Vasconcelos-Raposo e Rodrigues (2006) com o propósito de analisar o bem-estar psicológico, a auto-estima e a depressão em indivíduos praticantes de actividade física regular, questionaram 110 indivíduos. Verificaram que metades destes acreditavam que a actividade física pode contribuir para um melhor bem-estar psicológico; sendo estes resultados mais acentuados nas mulheres, nos idosos e nos atletas. Deste modo, os indivíduos optam pelo exercício físico como estratégia para regular os seus estados depressivos e de forma a aumentarem os seus níveis de energia.

Os tratamentos de depressão são, frequentemente, desenvolvidos por profissionais clínicos tais como: médicos de clínica geral, psiquiatras e psicólogos, recorrendo a aconselhamento e anti-depressivos (Teychenne, Ball, \& Salmon, 2008).

Estes últimos autores com o objectivo de estudar a relação entre a duração, a frequência e a intensidade da actividade física e a depressão observaram que existe uma associação inversa entre actividade física e a probabilidade dos adultos sofrerem de depressão. Com o mesmo propósito, Brown, Fond, Burton, Marshall e Dobson 
(2005), num estudo que contou com a participação de 9207 mulheres de meia-idade, constataram que existe uma clara relação entre actividade física e a diminuição de sintomas depressivos. O exercício ajuda a reduzir os sintomas depressivos consequentes dos acidentes vasculares cerebrais, contribuindo para uma melhoria na qualidade de vida destes indivíduos (Lai, Studenski, Richards, Perera, Reker, Rigler et al., 2006).

Fukukawa, Nakashima, Tsuboi, Kozakai, Doyo, Niino et al. (2004), examinaram a associação entre a actividade física e os sintomas depressivos em 1151 adultos no Japão, verificando que actividade física exerce um efeito protector no surgimento de sintomas depressivos.

Mais tarde, também Harris, Cronkite e Moos (2006) examinaram a relação entre actividade física, estratégias de exercício e depressão em pacientes inicialmente deprimidos. Os resultados sugeriram que a actividade física está associada à redução da depressão, ajudando a resolver problemas de saúde e as situações stressantes do quotidiano. Um estudo que comparou o efeito de dois programas de exercício, estruturado clinicamente e actividades domésticas, em 32 mulheres depressivas na área de Boston, concluíu que ambos estavam associados à redução de sintomas depressivos e que o programa das actividades domésticas aumentava o envolvimento das mulheres na prática desportiva (Craft, Freund, Culpepper, \& Perna, 2007).

Com o objectivo de estudar se o exercício regular está associado à ansiedade, depressão e personalidade em função da idade e do género numa população de 19288 indivíduos, Moor, Beem, Stubbe, Boomsma e Geus (2006) verificaram que os praticantes de exercício regular encontram-se em média menos deprimidos, menos neuróticos, menos ansiosos, mais extrovertidos e procuravam mais emoção e aventura, sendo mais desinibidos que os não praticantes. Hales, Dishman, Pfeiffer, Felton, Saunders Hales et al. (2006) testaram a relação entre auto-conceito, autoestima, actividade física e sintomas depressivos, em 1250 adolescentes, sendo que os resultados mostraram evidências de que a actividade física e a participação desportiva podem reduzir o risco de sintomas depressivos nas adolescentes.

Babyak, Blumenthal, Herman, Khatri, Doraiswamy, Moore e colaboradores (1999) avaliaram o estado da perturbação depressiva major em 156 adultos no final da realização de três tipos de actividades: exercício aeróbio, terapia farmacológica ou a combinação dos dois. Concluíram que após 4 meses de tratamento, os três grupos apresentavam melhorias significativas. Após 10 meses, o grupo do exercício aeróbio apresentou taxas de recaídas significativamente mais baixas do que no grupo dos indivíduos medicados. A terapia do exercício está associada, significativamente, ao benefício terapêutico de indivíduos com perturbação depressiva major, 
especialmente se este for contínuo e duradouro. Mais tarde, um estudo mostrou que, apesar de não existir um efeito anti-depressivo directo do exercício físico sobre a depressão, o facto de treinar envolveu os indivíduos num programa de tratamento cognitivo-comportamental, associado a mudanças positivas no funcionamento dos indivíduos e aumentando as estratégias, os esforços para continuar a praticar e melhorando a consciência de bem-estar físico (Vliet, Auweele, Knapen, Rzewnicki, Onghena, \& Coppenolle, 2004)

Juarbe, Gutiérrez, Gilliss e Lee (2005) investigaram as mudanças e a associação entre os sintomas depressivos, actividade física e índice de massa corporal, concluindo que os sintomas depressivos, o aumento de peso e a inactividade física em 232 mulheres na pré-menopausa requerem intervenções focalizadas devido às alterações hormonais que ocorrem nesta fase. Deste modo, a promoção de altos níveis de actividade física e a identificação dos riscos de depressão pode trazer melhorias significativas na saúde mental e física. McLarem, Beck, Patten, Fick e Adair (2008) estudaram a relação entre 0 índice de massa corporal e a saúde mental numa população adulta com indivíduos entre 18 e os 64 anos com cerca de 5383 indivíduos. Constataram que é importante considerar o tipo de doença mental, nível de severidade relativamente ao IMC, sexo e idade ao examinar a relação entre o IMC e a saúde mental. Do estudo constataram não existir uma associação global entre estas 2 variáveis, no entanto, constataram uma diversidade de associações aquando de uma análise detalhada das várias doenças ao nível da severidade.

Num estudo que contou com a participação de 4655 mulheres com idades compreendidas entre os 40 e 65 anos, estudou-se a associação entre a obesidade e sintomas depressivos através de uma população epidemiológica, observando-se que a obesidade e a depressão se encontram positivamente associadas (Linde, Jeffery, Finch, Simon, Ludman, Operskalski et al., 2007).

Na mesma perspectiva, Dragan e Akhtar-Danesh (2007), estudaram a relação entre o índice de massa corporal e depressão numa amostra de 12376 indivíduos, verificandose uma forte associação entre estas duas variáveis e diferenças de género. Nos homens, altos níveis de índice de massa corporal encontram-se associados a níveis severos de depressão, e ainda que, altos níveis de depressão encontram-se associados a índices de massa corporal baixos. Na maior parte das mulheres, não se verificou uma relação directa, nem estatisticamente significativa, entre obesidade e depressão. De realçar, a relação inversa entre a actividade física e o índice de massa corporal que se traduz na expressão "mais actividade física - menos problemas de peso".

No sentido de estudar a depressão em áreas rurais e urbanas, Colla, Buka, Harrington e Murphy (2006) constataram que a depressão era mais baixa nas áreas rurais da 
Nigéria e mais alta nos sujeitos residentes nas áreas urbanas dos Estados Unidos. As mulheres, com idades entre os 45 anos, que residem desde a infância, em meio urbano apresentam níveis de depressão mais altos. Hauenstein, Petterson, Rovnyak, Merwin, Heise e Wagner (2007), investigaram os tratamentos mentais facultados em meio rural e urbano. Os resultados evidenciaram que os residentes em áreas rurais recebem menos tratamentos de saúde mental quando comparados com indivíduos de áreas metropolitanas, numa amostra de 108983 sujeitos, com idade superior a 18 anos.

Em síntese, pela análise dos artigos revistos, constatamos que existe um consenso sobre a temática pois os estudos apontam para a existência de relações entre o exercício físico e a depressão e entre o IMC e a depressão, enquanto a relação conjunta entre estas três dimensões tem sido algo negligenciado na literatura.

Deste modo, este trabalho tem por objectivo conhecer a relação entre a depressão, o exercício físico e o IMC. Pretende-se ainda, compreender a diferença entre os praticantes de exercício físico e os não praticantes ao nível de sintomatologia depressiva; comparar o nível de depressão nos indivíduos que praticam os níveis de actividade física recomendado (no adulto, 30 minutos consecutivos ou fraccionados diariamente em cinco ou mais dias por semana, Haskell et al., 2007; Pate et al., 1995); compreender a relação entre os indivíduos que habitam em meio rural ou urbano e os níveis de depressão; e, por último, entender a relação entre os níveis de depressão e o índice de massa corporal da amostra recolhida.

\section{Metodologia}

\section{Amostra}

A amostra deste estudo foi constituída por 175 sujeitos. Inicialmente foram aplicados 200 questionários, mas 25 destes foram anulados, pois verificou-se que não eram válidos por ausência de resposta ou por incompreensão da resposta assinalada.

Deste modo, este estudo envolveu 43 elementos do sexo masculino $(24,6 \%)$ e 132 elementos do sexo feminino $(75,4 \%)$ dos distritos de Bragança, Porto e Vila Real, com idades compreendidas entre os 18 e os 27 anos de idade (20.95 \pm 2.27 ). Quanto ao estado civil, $97,7 \%$ dos indivíduos eram solteiros e $2,3 \%$ eram casados. No que diz respeito às habilitações literárias, o ensino secundário predomina com $61,7 \%$ do total da amostra enquanto os restantes $38,3 \%$ têm uma formação superior. Relativamente à profissão, a totalidade da amostra era estudante. 
Quadro 1: Caracterização da amostra

\begin{tabular}{lcc}
\hline & $n$ & $\%$ \\
\hline Sexo & 43 & 24,57 \\
Masculino & 132 & 75,43 \\
Feminino & & \\
Estado civil & 171 & 97,70 \\
Solteiro & 4 & 2,30 \\
Casado & & 61,71 \\
Habilitação literária & 108 & 38,29 \\
Ensino Secundário & 67 & \\
Ensino Superior & & \\
\hline
\end{tabular}

Relativamente à prática de actividade física (AF), as recomendações actuais envolvem o mínimo de 30 minutos consecutivos ou fraccionados em cinco ou mais dias da semana (Haskell et al., 2007; Pate et al., 1995). Perante o exposto, na nossa amostra constata-se que $13,1 \%$ da amostra $(n=23)$ atinge as recomendações, enquanto $86,9 \%$ $(n=152)$ não atinge. Quando diferenciados por níveis de $A F$, cerca de $26,9 \%$ são inactivos, 36,6\% praticam entre 1 a 2 dias por semana ("insuficientemente activos"), $23,4 \%$ foram considerados "moderadamente activos" ( 3 a 4 vezes por semana) e os $13,1 \%$ que atingiam as recomendações foram considerados como "muito activos"

\section{Procedimentos}

Utilizámos o Beck Depressive Inventory (Beck, Ward, Mendelson, Mock, \& Erbaugh, 1961; BDI), adaptado para língua portuguesa na versão do Brasil por Gorestein e Andrade (1998), que por sua vez foi adaptado para português de Portugal por Pinto e Vasconcelos-Raposo (no prelo) para avaliar a depressão e sintomas depressivos. Este inventário é constituído por 21 itens sendo que cada item contém 4 frases, que descrevem 4 possibilidades ordenadas por gravidade depressão sintomática com cotação de o a 3. No presente estudo, as respostas foram dadas numa escala de tipo Likert, representando 1 - "discordo", 2- "nem concordo nem discordo" e 3 "concordo". Os itens referem-se a tristeza, pessimismo, sensação de fracasso, falta de satisfação, sensação de culpa, sensação de punição, auto-depreciação, autoacusação, ideias suicidas, crises de choro, irritabilidade, retraç̧ão social, indecisão, distorção da imagem corporal, inibição no trabalho, distúrbio de sono, perda de apetite, perda de peso, preocupação somática, diminuição de libido. De acordo com Pinto e Vasconcelos-Raposo (no prelo), a BDI apresenta uma estrutura factorial com 3 constructos. O factor 1 representa a dimensão auto-depreciação e inclui os itens 1, 4, 12,13 e 14; o factor 2 designado cognição-afecto é constituído pelos itens 3, 5, 6, 7, 15 
e 17; e o factor 3 representa a dimensão somática e engloba os itens 16,18, 19 e 21. Aquando da utilização destes factores para a amostra em estudo, verificou-se que os coeficientes alpha de Cronbach foram muito baixos, isto é 0.54 para os factores 1 e 2 e 0.28 para o factor 3 . Tendo em conta estes valores de consistência interna medíocres e ainda, o facto da dimensão depressão global (somatório total dos itens) apresentar um valor de consistência interna de 0.79, decidiu-se tratar esta variável unidimensionalmente, visto este atingir um valor satisfatório.

Utilizaram-se também, duas variáveis para avaliar a frequência do exercício físico habitual numa semana normal e na última semana, com base numa escala intervalar que varia entre zero e sete dias. Para isso efectuámos uma adaptação da escala de exercício físico de Prochaska, Sallis e Long (2001), apresentando a duração de 30 minutos como valor mínimo de prática de AF por dia.

\section{Resultados}

Relativamente à análise inicial descritiva (dados não apresentados) verificou-se que o valor de skewness (assimetria da curva) para a depressão geral encontra-se dentro do intervalo considerado para uma distribuição normal; quanto ao valor de kurtosis (achatamento da curva), este encontra-se ligeiramente acima do intervalo, porém tendo em conta a dimensão da nossa amostra, foram estudados os dados como seguindo uma distribuição normal, recorrendo à estatística paramétrica.

No quadro 2 são apresentados os resultados de análises comparativas, onde se constata que o sexo feminino e os indivíduos que não atingem as recomendações de AF reportam níveis médios superiores de depressão, sendo essas diferenças significativas $(p<0.05)$.

Quadro 2. Análise comparativa por sexo, recomendações de AF e local de residência

\begin{tabular}{cccc}
\hline & Depressão Geral & $t$ & $p$ \\
\hline Feminino & $6.48(0.50)$ & 2.59 & 0.01 \\
Masculino & $6.24(0.55)$ & 2.65 & 0.01 \\
\hline Não atinge & $6.46(0.52)$ & & 0.10 \\
Atinge & $6.16(0.44)$ & 1.40 & \\
\hline Rural & $6.37(0.51)$ & $6.48(0.52)$ & \\
Urbano & & & \\
\hline
\end{tabular}

Da análise comparativa das categorias da prática de exercício físico, constatou-se que a depressão não apresenta valores significativos ( $p>0.05$ ); os valores médios vão diminuindo à medida que se passa para uma categoria em que o sujeito é mais activo 
no que diz respeito à prática desportiva: "inactivo" (6.42 \pm 0.53$)$, "insuficientemente activo" (6.36 \pm 0.61$)$, "moderadamente activo" (6.37 \pm 0.51$)$ e "muito activo" (6.08 \pm 0.48$)$.

A análise comparativa das categorias do IMC, revelou que a depressão geral não apresenta valores significativos ( $p>0.05$ ), mas apesar disso, os valores médios tendem a diminuir à medida que se passa para uma categoria em que o IMC é superior:

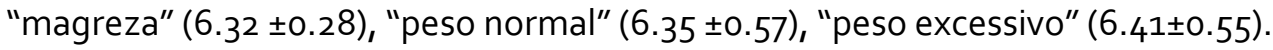

Quadro 3. Análise dos participantes por categoria e dos valores médios (ANOVA) das categorias da frequência de prática de exercício físico, e do IMC

\begin{tabular}{lcccc}
\hline & $N$ & $\begin{array}{c}\text { Depressão } \\
\text { Geral }\end{array}$ & $F$ & $p$ \\
\hline Inactivo & 47 & $6.42(0.53)$ & & \\
Insuficientemente activo & 64 & $6.36(0.61)$ & 2.062 & 0.107 \\
Moderadamente activo & 41 & $6.37(0.51)$ & & \\
Muito activo & 23 & $6.08(0.48)$ & & \\
\hline Magreza & 11 & $6.32(0.28)$ & & \\
Peso Normal & 146 & $6.35(0.57)$ & & \\
Peso Excessivo & 18 & $6.41(0.55)$ & & \\
\hline
\end{tabular}

De acordo com os valores apresentado no quadro 3, quanto ao índice de massa corporal, constatamos que $6,3 \%(n=11)$ da amostra encontra-se no intervalo da magreza (IMC $<18.5), 83,4 \%$ dentro do peso normal (IMC 18.5><25)e 10,3\% ( $=18$ ) com peso excessivo (IMC>25).

\section{Discussão}

De acordo com os nossos resultados, a depressão e o exercício físico são duas variáveis que apresentam uma relação inversa. Quando uma das variáveis aumenta, a outra tende a diminuir significativamente. Esta associação já tinha sido apontada por Teychenne e colaboradores (2008), Brown e colaboradores (2005), Lai e colaboradores (2006), Fukukawa e colaboradores (2004), Harris e colaboradores (2006), Hales e colaboradores (2006), Babyak e colaboradores (1999) que concluíram que a prática de exercício físico contribui para a diminuição dos sintomas depressivos.

Apesar da relação entre a depressão e o IMC não sugerir valores significativos, convém referir que o comportamento das médias sugere um aumento das médias da variável depressão conforme cresce o IMC. Mc Larem e colaboradores (2008) também 
tinham verificado a inexistência de relação estatisticamente significativa entre a depressão geral e o IMC. Porém, chamavam atenção para o facto de se ter em conta outros factores como o nível de severidade relativamente ao IMC, a idade e o tipo de doença mental no qual se verificavam associações. No entanto, a nossa amostra para cada categoria do IMC não apresenta dimensões que permitam a comparação, a fim de obter resultados generalizáveis à população portuguesa. Contudo, Linde e colaboradores (2007) tinham observado uma associação positiva entre a depressão e a obesidade, o que não é verificado no presente estudo. É de realçar que $83.4 \%$ da nossa amostra apresentou peso normal, enquanto $10.3 \%$ apresenta peso excessivo e $6.3 \%$ magreza. Estes valores, de algum modo vão ao encontro dos argumentos apresentados pelo grupo de trabalho da APA (American Psychological Association) que participa no processo de redefinição dos critérios de diagnóstico das perturbações alimentares a serem implementados com o DSM-V, previsto para 2012.

Num estudo deste tipo, com populações clinicamente diagnosticadas, importa ter em consideração a terapêutica medicamentosa a que os sujeitos possam estar submetidos, pois é necessário controlar os efeitos de uma relação positiva entre a ingestão de medicamentos e o IMC.

Hauenstein e colaboradores (2007) observaram que os residentes nas áreas rurais recebem menos tratamentos de saúde mental em comparação com os residentes em áreas metropolitanas. No mesmo sentido. Colla e colaboradores (2006) mostraram que a depressão era mais baixa nas áreas rurais da Nigéria do que nas áreas urbanas dos Estados Unidos da América. Os resultados do nosso estudo vão ao encontro do que se verifica na literatura internacional, assim como se aplicam os argumentos de carácter sociocultural, avançados naquele estudo, pois as desigualdades políticoecómicas que explicam a desigualdade de acesso a serviços de saúde, existentes entre as zonas rurais e urbanas, são também verdade na realidade por nós estudada. Porém, deve-se ter em conta que a nossa amostra foi constituída apenas por estudantes universitários, e apesar de serem provenientes do meio rural, a vida académica envolve-os, maioritariamente, num contexto urbano, ou seja, os habitantes que se indentificaram como residentes no meio rural não são verdadeiramente representantes dessa zona de residência, uma vez que já foram aculturados ao ethos da vida urbana e em particular da vida inerente à condição de estudante universitário.

De acordo com os nossos resultados, a depressão é, em média, superior nos indivíduos que não atingem os níveis de exercício físico recomendado em relação aos que atingem os níveis recomendados. O nosso estudo vai ao encontro das conclusões de Moor e colaboradores (2006) tendo em conta que os autores verificaram que em 
média os praticantes de exercício regular encontravam-se menos ansiosos, menos deprimidos, mais extrovertidos e desinibidos, procurando mais a emoção e a aventura. Também constatamos que os valores médios de depressão diminuem à medida que se passa para uma categoria do exercício físico no qual o indivíduo é mais activo, podendo-se concluir que o exercício físico contribui, significativamente, na redução ou prevenção da depressão ou sintomas depressivos.

A depressão e o IMC não apresentam uma associação significativa, embora os valores médios tendem a diminuir e chegam mesmo a ser inferiores para os indivíduos com peso excessivo, enquanto o valor médio superior se verifica para a categoria "magreza", mas como referimos anteriormente, a dimensão da nossa amostra não permite retirar conclusões adequadas pelas categorias do IMC. Pelo contrário, Dragan e colaboradores (2007) tinham verificado uma forte associação entre o IMC e a depressão, sendo que altos níveis de depressão estavam associados a baixos e altos níveis de IMC nos homens. No caso das mulheres não se verificaram diferenças estatisticamente significativas.

Em média, as mulheres apresentam valores superiores de depressão quando comparados com os homens, o que confirma o estudo de Campos e Gonçalves (2004) realizado em Portugal, e igualmente com uma amostra de estudantes universitários. Aquando da comparação dos níveis de exercício físico com as diferentes categorias de depressão verificou-se que o exercício físico tem um efeito de prevenção ao nível da sintomatologia depressiva, porém, no nosso estudo, não se apresentou estatisticamente significativo. Já anteriormente, Teychenne e colaboradores (2008) tinham observado uma associação estatisticamente significativa quanto ao efeito preventivo da actividade física sobre a depressão.

\section{Conclusão}

Os nossos resultados demonstram que à medida que se aumenta a prática de exercício físico, os sintomas depressivos tendem a diminuir, não se verificando nenhuma associação entre a depressão e o IMC geral. Porém verificou-se que os indivíduos que praticam os níveis de exercício físico recomendado apresentam níveis médios de depressão inferiores aos que não atingem. De notar que os estudantes universitários do sexo feminino apresentam valores médios superiores de depressão em comparação com o sexo masculino. Constatámos que o que a prática desportiva poderá ter um carácter positivo na prevenção ou redução da depressão nos adultos.

Em investigações futuras, sugerimos a realização de estudos que procurem comparar o efeito de programas de actividade na melhoria dos estados de humor devendo ser 
preocupação identificar as diferenças entre as actividade de carácter aeróbio e anaeróbio, que poderá ter efeitos mais eficazes na melhoria da depressão.

A literatura existente está, fundamentalmente, dirigida para a população idosa levando-nos a alertar para que futuras investigações sobre esta problemática incidam nas faixas etárias de adolescentes e adultos.

\section{Bibliografia}

Associação Americana de Psiquiatria (2000). Manual de Diagnóstico e Estatística das Perturbações Mentais ( $4^{\mathrm{a} E d i c ̧ a ̃ o) . ~ L i s b o a: ~ C l i m e p s i . ~}$

Babyak, M., Blumenthal, J., Herman, S., Khatri, P., Doraiswamy, M., Moore., K. et al. (1999). Exercise treatment for major depression: Maintenance of therapeutic benefit at 10 months. Psychosomatic Medicine, 62, 633-638.

Beck, A.T., Ward, C., Mendelson, M., Mock, J. \& Erbaugh, J. (1961). An inventory for measuring depression. Archives of General Psychiatry, 4, 561-571.

Brown, W., Fond, J., Burton, N., Marshall, A., \& Dobson, A. (2005) Prospective study of physical activity and depressive symptoms in middle-aged women. American Journal of Preventive Medicine, 29 (4), 265-272.

Buckworth, J. \& Dishman, R. (2002). Exercise Psychology. Champaign: Human Kinetics.

Campos, R. \& Gonçalves, B. (2004) Alguns dados sobre a prevalência de sintomatologia depressiva na população universitária portuguesa. In C. Machado, L. Almeida, M. Gonçalves e V. Ramalho (Eds.), Actas da X Conferência Internacional de Avaliação Psicológica: Formas e contextos (pp. 50-53). Braga: Psiquilibrios Edições.

Colla, J., Buka, S., Harrington, D. \& Murphy, J. (2006). Depression and modernization. Social Psychiatry and Psychiatric Epidemiology, 41, 271-279.

Craft, L., Freund, K., Culpepper, L., \& Perna, F. (2007) Intervention study of exercise for depressive symptoms in women. Journal of Women's Health, 16 (10), 1499-1509.

Dragan, A. \& Akhtar-Danesh, N. (2007). Relation between body mass index and depression: A structural equation modeling approach. Medical Research Methodology, 7 (17), 1-8.

Fukukawa, Y., Nakashima, C., Tsuboi, S., Kozakai, R., Doyo, W., Niino, N. et al. (2004) Age differences in the effect of physical activity on depressive symptoms. Psychology and Aging, 19 (2), 346-351.

Goreinstein, C. \& Andrade, L. (1998). Inventário de depressão de Beck: Propriedades psicométricas da versão em português. Revista de Psiquiatria Clínica, 25 (5), 245-250.

Hales, D., Dishman, R., Pfeiffer, K., Felton, G., Saunders, R., Hales, D. et al. (2006). Physical self-concept and self-esteem mediate cross-sectional relations of physical activity and sport participation with depression symptoms among adolescent girls. Health Psychology, 25 (3), 396-407.

Harris, A., Cronkite, R., \& Moos, R. (2006). Physical activity, exercise coping and depression in a 10- year cohort study of depressed patients. Journal of Affective Disorders, 93, 79-85.

Haskell, W.L., Lee, I., Pate, R.R., Powell, K.E., Blair, S.N. et al. (2007). Physical activity and public health: Updated recommendation for adults from the American College of Sports 
32 | Relação entre exercício físico, depressão e índice de massa corporal

Medicine and the American Heart Association. Medicine \& Science in Sports \& Exercise, 39 (8), 1423-1434.

Hauenstein, E., Petterson, S., Rovnyak, V., Merwin, E., Heise, B., \& Wagner, D. (2007). Rurality and mental health treatment. Administration Policy in Mental Health \& Mental Health Services Research, 34, 255-267.

Juarbe,T., Gutiérrez, Y., Gilliss, C., \& Lee, K. (2005). Depressive symptoms, physical activity and weight gain in premenopausal latina and white women. The European Menopausal Journal, 55, 116-125.

Lai, S., Studenski, S., Richards, L., Perera, S., Reker, D., Rigler, S. et al. (2006). Therapeutic exercise and depressive symptoms after stroke. The American Geriatrics Society, 54, 240247.

Linde, J., Jeffery, R., Finch, E., Simon, G., Ludman, E., Operskalski, B. et al. (2007). Relation of body mass index to depression and weighing frequency in overweight women. Preventive Medicine, 45, 75-79.

McKendree-Smith, N.L., Floyd, M., \& Scogin, F. (2003). Self-administraded treatments for depression: A review. Journal of Clinical Psychology, 59 (3), 275-288.

McLarem, L., Beck, C., Patten, S., Fick, G., \& Adair, C. (2008). The relationship between body mass index and mental health: A population-based study of the effects of the definition of mental health. Social Psychiatry and Psychiatric Epidemiology, 43, 63-71.

Moor, M., Beem, A., Stubbe, J., Boomsma, D., \& De Geus, E. (2006). Regular exercise, anxiety, depression and personality: A population-based study. Preventive Medicine, 42, 273-279.

Paluska, S.A., \& Schwenk, T.L., (2000). Physical activity and mental health. Sports Medicine, 29, 167-180.

Pate, R., Pratt, M., Blair, S., Haskell, W., Macera, C., Bouchard, C. et al. (1995). Physical activity and public health: A recommendation from the Centers for Disease Control and Prevention and the American College of Sports Medicine. The Journal of the American Medical Association, 273 (5), 402-407.

Pinto, G. \& Vasconcelos-Raposo, J. (no prelo). Validação do Inventário de depressão de Beck como instrumento de pesquisa em estudantes universitários portugueses. Manuscrito não publicado.

Prochaska, J., Sallis, J., \& Long, B. (2001). A physical activity screening measure for use with adolescents in primary care. Archives of Pediatric Adolescence Medicine, 155, 554-599.

Teychenne, M., Ball, K. \& Salmon, J. (2008). Physical activity and likelihood of depression in adults: A review. Preventive Medicine, 46, 397-411.

Toker, S., Shinom, A., \& Melamed, S. (2008) Depression and the metabolic syndrome: Genderdepender associations. Depression and Anxiety, 25, 661-669.

Vasconcelos-Raposo, J. \& Rodrigues, A. (2006). A relação entre o bem-estar psicológico, a autoestima, a depressão e a religiosidade entre praticantes de actividade física regular e não praticantes em idosos. Monografia não publicada, Vila Real: UTAD.

Vliet, P., Auweele, Y., Knapen, J., Rzewnicki, R., Onghena, P. \& Coppenolle. H. (2004). The effect of fitness training on clinically depressed patients: An intra-individual approach. Psychology of Sport and Exercise, 5, 153-167. 